\title{
Número de dias e de graus-dia entre a iniciação e a diferenciação da panícula em cultivares de arroz irrigado
}

\author{
Number of days and degree-days between panicle initiation and \\ panicle differentiation in irrigated rice cultivars \\ Cristiano de Carli ${ }^{\text {I }}$ Silvio Steinmetz ${ }^{\text {II }}$ Nereu Augusto Streck ${ }^{\text {III }}$ \\ Enio Marchesan ${ }^{\text {III }}$ Michel Rocha da Silva ${ }^{\text {IV }}$
}

\section{RESUMO}

O objetivo deste trabalho foi determinar o número de dias e de graus-dia em que a iniciação da panícula (IP) antecede a diferenciação da panícula (DP) para cultivares de arroz de diferente duração de ciclo. Um experimento de campo foi conduzido em área de várzea, em Santa Maria, RS, e foi composto de três datas de semeadura e quatro cultivares ('IRGA 421', 'BRS Querência', 'IRGA 424'e 'SCSBRS Tio Taka') em dois anos agrícolas (2011/12 e 2012/13), no delineamento de blocos acaso, com quatro repetições. A data de ocorrência da DP foi estimada a partir das tabelas contidas em STEINMETZ et al. (2010) e, aproximadamente 14 dias antes da data estimada da DP, foram realizadas amostragens diárias de 10 plantas para determinar a IP em laboratório sob lupa de aumento de 45 vezes. Após a data de ocorrência da IP, 10 plantas foram coletadas diariamente para a análise da DP através do "ponto de algodão". A soma térmica foi calculada considerando a temperatura basal de $11^{\circ} \mathrm{C}$. As maiores diferenças entre as datas de ocorrência da IP e DP foram de 8 e 6 dias, e de 116,4 e $73,7^{\circ} \mathrm{C}$ dia. Na maior parte dos resultados, as diferenças variaram entre 2 e 8 dias, e entre 23,8 e $116,4^{\circ} \mathrm{C}$ dia. Concluiu-se que o periodo máximo que a IP antecede a DP é de oito dias, correspondendo a $116,4^{\circ} \mathrm{C}$ dia, não sendo influenciado pela época de semeadura nem pelo comprimento de ciclo das cultivares.

Palavras-chave: soma térmica, nitrogênio em cobertura, Oryza sativa, desenvolvimento vegetal.

\section{ABSTRACT}

The objective of this study was to determine the number of days and degree-days in which panicle initiation (PI) takes place before panicle differentiation (PD) for rice cultivars with different developmental cycle duration. A field experiment was carried out in a lowland area, in Santa Maria, RS, Brazil. The experiment consisted of three sowing dates and four rice cultivars ('IRGA 421', 'BRS Querência', 'IRGA 424' and 'SCSBRS Tio Taka') during two growing seasons (2011/12 and 2012/13), in a complete randomized block design with four replications. The date of PD was estimated from the tables contained in STEINMETZ et al. (2010) and around 14 days before the estimated date of PD. Ten plants were sampled daily to determine PI in a laboratory under a 45 times magnifying glass. After PI, 10 plants were sampled daily to determine PD using the "cotton ball" stage as a marker. Thermal sum was calculated considering the basal temperature of $11^{\circ} \mathrm{C}$. The greatest differences between the dates of occurrence between PI and PD were 8 and 6 days, and 116.4 and $73.7^{\circ} \mathrm{C}$ day. Most of the differences varied between 2 and 8 days, and between 23.8 and $116.4^{\circ} \mathrm{C}$ day. It was concluded that the maximum period that PI takes place before the PD is eight days, corresponding to $116.4^{\circ} \mathrm{C}$ day, being influenced neither by sowing date nor by the length of cultivars cycle.

Key words: thermal time, nitrogen topdressing, Oryza sativa, plant development.

\section{INTRODUÇÃO}

O arroz (Oryza sativa L.) é uma poaceae, aquática, de cultivo anual (YOSHIDA, 1981), com produção mundial estimada pela FAO (2014) em 500,7 milhões de toneladas de arroz moído. A produção brasileira é de 12,77 milhões de toneladas de arroz em casca, sendo a região Sul a principal produtora (9,61 milhões de toneladas). A estimativa para 2014 é que o Rio Grande do Sul alcance a

ICurso de Agronomia, Universidade Federal de Santa Maria (UFSM), Santa Maria, RS, Brasil.

"Embrapa Clima Temperado, Pelotas, RS, Brasil.

IIIDepartamento de Fitotecnia, Universidade Federal de Santa Maria (UFSM), 97105-900, Santa Maria, RS, Brasil. E-mail: nstreck2@yahoo.com.br. Autor para correspondência.

${ }^{\text {IV}}$ Programa de Pós-graduação em Agronomia, Universidade Federal de Santa Maria (UFSM), Santa Maria, RS, Brasil. 
produtividade de $7.574 \mathrm{~kg} \mathrm{ha-1}$, com produção de 8,38 milhões de toneladas (CONAB, 2014). Devido à grande importância econômica e alimentar do arroz e apesar da elevada produtividade atual das lavouras arrozeiras gaúchas, acredita-se que a produtividade ainda possa ser aumentada com a otimização de alguns aspectos relacionados ao manejo da cultura.

Dentre os aspectos, destaca-se a adubação nitrogenada, um dos fatores determinantes na produtividade do arroz. Fertilizantes fornecedores de nitrogênio $(\mathrm{N})$ são, normalmente, mais caros, por possuir alta interação com as condições ambientais como o clima, a fertilidade do solo, manejo de doenças e pragas e pelo manejo da água, tornando a eficiência agronômica dessa prática muito variável. Portanto, a SOSBAI (2012) recomenda aplicação de $\mathrm{N}$ em dois momentos, com vistas a aumentar a eficiência agronômica da adubação nitrogenada: uma em V3V4 (três a quatro folhas expandidas) e a segunda em R0 (iniciação da panícula).

No entanto, o R0, ou iniciação da panícula (IP), é de difícil identificação na planta, não sendo possível sua visualização a olho nu. Como indicador mais fácil de identificar a olho nu, usa-se como referência para a segunda adubação nitrogenada em cobertura (ANC) a diferenciação da panícula (DP), R1 da escala de COUNCE et al. (2000), conhecido como "ponto de algodão", que ocorre de três a cinco dias após a IP (STANSEL, 1975). O problema é que a data de ocorrência da DP é variável por ser dependente da temperatura (STANSEL, 1975; STEINMETZ et al., 2009). Por isso, é preferível estimar a data de ocorrência da DP usando-se o método de graus-dia, ou soma térmica, ao invés do número de dias pelo calendário civil (SLATON et al., 1996; STEINMETZ et al., 2010). Baseando-se nesse princípio, STEINMETZ et al. (2010) estimaram a data de ocorrência da DP, para seis subgrupos de cultivares, em 17 localidades do Rio Grande do Sul (RS), usando séries históricas (30 anos) de temperatura média diária do ar (Tm) e, posteriormente, disponibilizaram para consulta por meio do programa GD Arroz, disponível no endereço $<$ http://www.cpact.embrapa.br/agromet $>$ (STEINMETZ et al., 2014). Para aumentar a precisão do uso da DP como referência para aplicar a ANC na IP, como recomenda a SOSBAI (2012), é necessário que se conheça, para as cultivares utilizadas no Rio Grande do Sul, em quantos dias e quantos grausdia a IP antecede a DP, e esta informação ainda não é conhecida, constituindo-se na motivação para realização deste trabalho.

O objetivo neste trabalho foi determinar o número de dias e de graus-dia em que a iniciação da panícula (IP) antecede a diferenciação da panícula (DP) para cultivares de arroz de diferente duração de ciclo.

\section{MATERIAL E MÉTODOS}

O experimento de campo foi conduzido na área de várzea do Departamento de Fitotecnia da Universidade Federal de Santa Maria, em Santa Maria, RS, durante os anos agrícolas 2011/12 e 2012/13. O solo é classificado como Planossolo Háplico eutrófico arênico, pertencente à unidade de mapeamento Vacacaí. Os tratamentos foram três datas de semeadura em cada ano (17 de outubro, 18 de novembro e 19 de dezembro de 2011, no primeiro ano agrícola, e 19 de outubro, 18 de novembro de 2012 e 4 de janeiro de 2013, no segundo ano agrícola) e quatro cultivares, 'IRGA 421' (ciclo muito precoce), 'BRS Querência' (ciclo precoce), 'IRGA 424' (ciclo médio) e 'SCSBRS Tio Taka' (ciclo tardio), no delineamento blocos ao acaso, com quatro repetições. Cada repetição constou de uma parcela de 1,5 x 5,0m, com 9 linhas, distanciadas de $17 \mathrm{~cm}$. A densidade de plantas $\left(\mathrm{pl} \mathrm{m}{ }^{-2}\right)$ em 2011/12, após a emergência, foi de 288, 284, 276 e 173, e em 2012/13 foi de 160, 166, 169 e 123, respectivamente, para as cultivares 'IRGA 421', 'BRS Querência', 'IRGA 424' e 'SCSBRS Tio Taka'. As duas primeiras datas de semeadura estão dentro e a terceira está fora do zoneamento agrícola do arroz no Rio Grande do Sul (MAPA, 2013). Elas foram selecionadas para expor as plantas a diferentes condições meteorológicas durante o mesmo ano agrícola.

A data de ocorrência da diferenciação da panícula (DP), R1 da escala de COUNCE et al. (2000), foi inicialmente estimada a partir das tabelas contidas em STEINMETZ et al. (2010), baseandose na data de $50 \%$ de emergência das plântulas da parcela. Com base nessa estimativa, 14 dias antes da data estimada da DP, foram iniciadas as coletas diárias de 10 plantas selecionadas aleatoriamente visando determinar a data de ocorrência da IP (R0 da escala de COUNCE et al., 2000) e da DP.

Para a análise da IP, as plantas coletadas no campo foram levadas ao laboratório. A análise consistiu na dissecação do colmo principal, considerado o colmo com maior diâmetro, em cada uma das plantas amostradas. A identificação da IP foi realizada com uma lupa, com aumento de 45 vezes, e com base nos critérios do IRRI (2012). A partir da data de ocorrência da IP, 10 plantas foram coletadas diariamente para a análise da DP. Os colmos principais de cada uma das 10 plantas foram cortados longitudinalmente com uma lâmina de barbear e a DP foi identificada a olho nu pela presença do "ponto 
de algodão" (SOSBAI, 2012). Foi considerado que a cultivar estava em IP ou DP quando $50 \%$ das plantas (colmo principal) amostradas estavam no estádio.

Foi calculada a soma térmica acumulada (STA) da emergência até a IP e da emergência até a DP e, posteriormente, contabilizado o número de dias e a STA em que a IP antecede a DP. Para cálculo da STA, foram usados os dados de temperatura média diária do ar medidos na estação meteorológica do $8^{\circ}$ DISME/INMET, instalada a aproximadamente $500 \mathrm{~m}$ do experimento, com a equação:

$$
\mathrm{GD}=\sum_{\mathrm{i}=1}^{\mathrm{n}}(\mathrm{Tm}-\mathrm{Tb})
$$

em que GD $\left({ }^{\circ} \mathrm{C}\right.$ dia) representa os graus-dia acumulados ou a soma térmica acumulada (STA), obtido pelo somatório da diferença entre a temperatura média diária $(\mathrm{Tm})$ e a temperatura base $(\mathrm{Tb})$ de $11^{\circ} \mathrm{C}$ (INFELD et al., 1998), da emergência ( $\mathrm{i}=1)$ à $\mathrm{DP}(\mathrm{n})$ e entre a IP e a DP.

\section{RESULTADOS E DISCUSSÃO}

$\mathrm{Na}$ figura 1, estão fotos do ápice meristemático de plantas de arroz em IP tiradas sob a lupa e, na figura 2, uma foto do ápice meristemático do colmo principal de uma planta de arroz em DP. No ano agrícola 2011/12, as maiores diferenças entre as datas de ocorrência da iniciação e da diferenciação da panícula (IP-DP) foram de 8 e 6 dias, respectivamente, para a cultivar 'IRGA 421', semeada em 19 de dezembro, e, para a cultivar 'IRGA 424', semeada em 17 de outubro (Tabela 1). Já na safra 2012/13, as maiores diferenças foram de 6 dias nas semeaduras de 19 de outubro e de 4 de janeiro, ambas para a cultivar 'SCS BRS Tio Taka' (Tabela 2). Considerando a maioria das cultivares e datas de semeadura, as diferenças entre IP e DP variaram entre 2 e 5 dias, sendo semelhantes às obtidas por STANSEL (1975), indicando que a iniciação antecede a diferenciação da panícula entre 3 e 5 dias. Na média de todas as cultivares, em todas as épocas de semeadura, nas duas safras, a diferença DP-IP foi de 4 dias.

Quanto às somas térmicas, no ano agrícola 2011/12, as maiores diferenças entre as datas de ocorrência da IP e da DP foram de 116,4 e $73,7^{\circ} \mathrm{C}$ dia, respectivamente, para a cultivar 'IRGA 421', semeada em 19 de dezembro, e, para a cultivar 'IRGA 424', semeada em 17 de outubro (Tabela 1). Já na safra 2012/13, as maiores diferenças foram de 77,7 e $77,9^{\circ} \mathrm{C}$ dia na semeadura de 19 de outubro, para as cultivares 'IRGA 424' e 'SCS BRS Tio Taka' (Tabela 2). Considerando

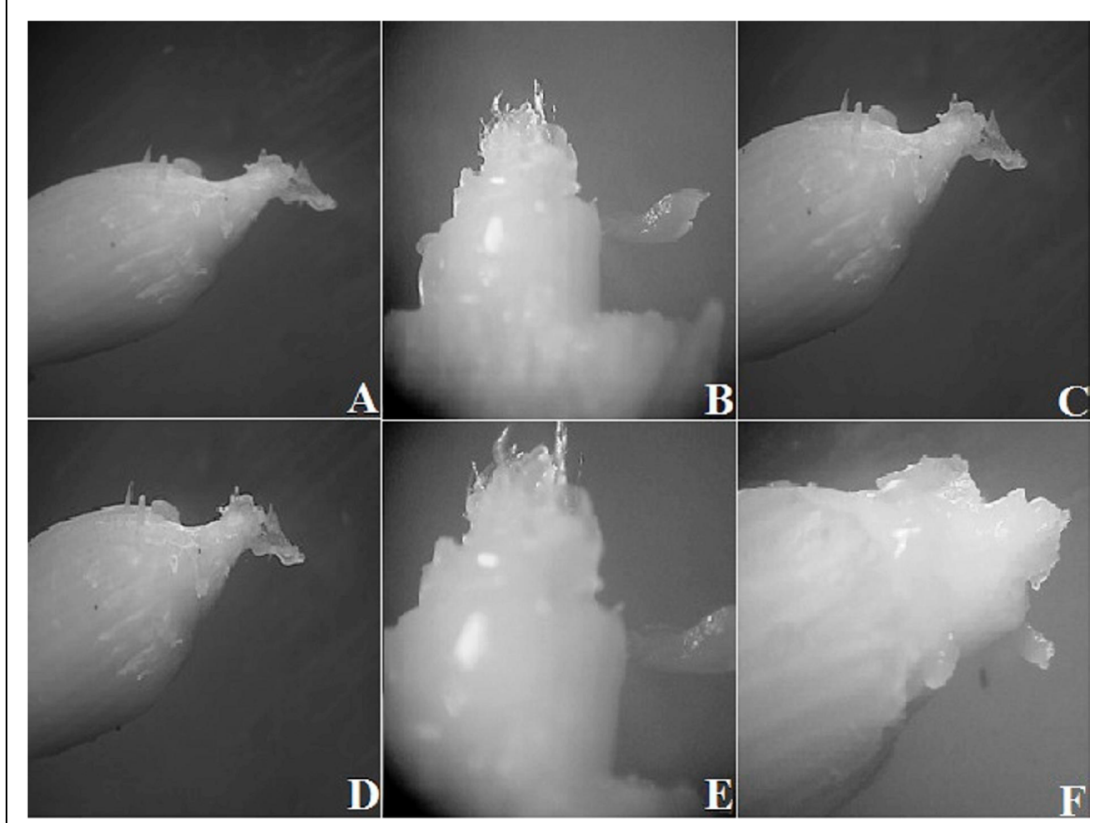

Figura 1 - Fotos do ápice meristemático de plantas de arroz em iniciação da panícula, R0 de COUNCE et al. (2000), tiradas com lupa de aumento de 45 vezes, durante o ano agrícola 2012/13. 


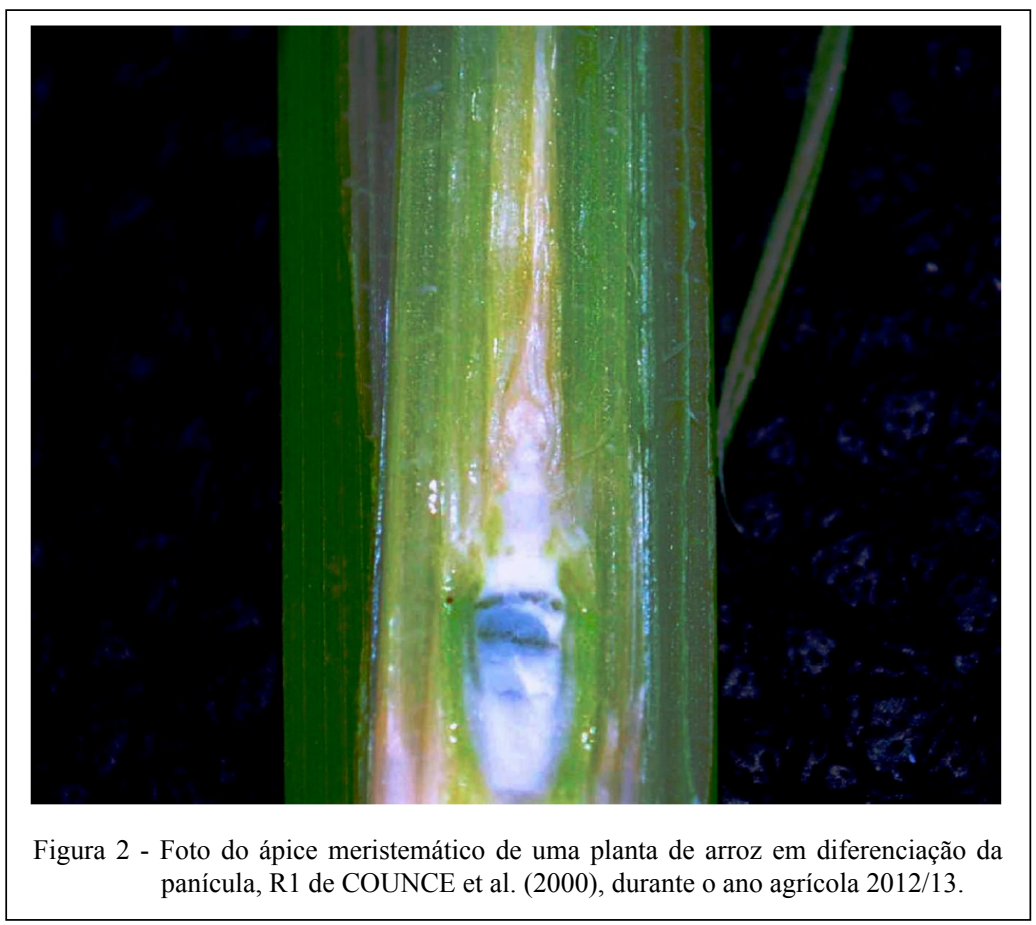

a maioria das cultivares e datas de semeadura, as diferenças entre IP e DP variaram entre 23,8 e $116,4^{\circ} \mathrm{C}$ dia. Na média de todas as cultivares, em todas as épocas de semeadura, nas duas safras, a diferença DP-IP foi de $53,1^{\circ} \mathrm{C}$ dia. Esse valor de STA é muito próximo de $48^{\circ} \mathrm{C}$ dia, que seria obtido pela multiplicação de 4 dias (média), em que a IP antecede a DP por $12^{\circ} \mathrm{C}$ dia. Este foi o valor médio diário de STA, da emergência à DP, obtido por STEINMETZ et al. (2010), para 16 cultivares de diferentes comprimentos de ciclo.
A hipótese inicial deste estudo era de que a diferença DP-IP poderia ser influenciada pela época de semeadura, pois, em geral, para a maioria das cultivares, há um retardo e um adiantamento na data de ocorrência da DP, respectivamente, para as semeaduras do cedo e do tarde (STEINMETZ et al., 2009). Da mesma forma, poderia se esperar que a diferença DP-IP fosse maior para as cultivares de ciclo tardio e médio, uma vez que a duração da fase vegetativa dessas cultivares é maior do que das cultivares de

Tabela 1 - Data de ocorrência da iniciação da panícula (IP), da diferenciação da panícula (DP), diferença entre as datas de DP e IP, em dias, soma térmica acumulada (STA) para atingir a DP e diferença de STA entre as datas de DP e IP, em ${ }^{\circ} \mathrm{C}$ dia.

\begin{tabular}{|c|c|c|c|c|c|c|c|}
\hline Cultivar & $\begin{array}{c}\text { Data de } \\
\text { Semeadura }\end{array}$ & $\begin{array}{c}\text { Data de } \\
\text { Emergência }\end{array}$ & IP & DP & $\begin{array}{l}\text { DP-IP } \\
\text { (dias) }\end{array}$ & $\begin{array}{c}\text { STA } \\
\left({ }^{\circ} \mathrm{C} \text { dia }\right)\end{array}$ & $\begin{array}{l}\text { DP-IP } \\
\left({ }^{\circ} \mathrm{C} \text { dia }\right)\end{array}$ \\
\hline 'IRGA 421' & $17 / 10 / 2011$ & $03 / 11 / 2011$ & - & $10 / 12 / 2011$ & - & 435,70 & - \\
\hline 'BRS Querência' & $17 / 10 / 2011$ & $03 / 11 / 2011$ & $16 / 12 / 2011$ & $20 / 12 / 2011$ & 4 & 558,40 & 55,30 \\
\hline 'IRGA 424' & $17 / 10 / 2011$ & $03 / 11 / 2011$ & $28 / 12 / 2011$ & $03 / 01 / 2012$ & 6 & 730,90 & 73,70 \\
\hline 'SCSBRS Tio Taka' & $17 / 10 / 2011$ & $03 / 11 / 2011$ & $19 / 01 / 2012$ & $23 / 01 / 2012$ & 4 & 1020,40 & 62,30 \\
\hline 'IRGA 421' & $18 / 11 / 2011$ & 29/11/2011 & $06 / 01 / 2012$ & $09 / 01 / 2012$ & 3 & 529,80 & 49,60 \\
\hline 'BRS Querência' & $18 / 11 / 2011$ & 29/11/2011 & $12 / 01 / 2012$ & $16 / 01 / 2012$ & 4 & 624,50 & 45,80 \\
\hline 'IRGA 424' & $18 / 11 / 2011$ & 29/11/2011 & $19 / 01 / 2012$ & $23 / 01 / 2012$ & 4 & 730,50 & 62,30 \\
\hline 'SCSBRS Tio Taka' & $18 / 11 / 2011$ & 29/11/2011 & $14 / 02 / 2012$ & $17 / 02 / 2012$ & 3 & 1102,00 & 53,30 \\
\hline 'IRGA 421' & $19 / 12 / 2011$ & $27 / 12 / 2011$ & $25 / 01 / 2012$ & $02 / 02 / 2012$ & 8 & 536,60 & 116,40 \\
\hline 'BRS Querência' & $19 / 12 / 2011$ & $27 / 12 / 2011$ & $07 / 02 / 2012$ & $10 / 02 / 2012$ & 3 & 656,20 & 44,20 \\
\hline 'IRGA 424' & $19 / 12 / 2011$ & $27 / 12 / 2011$ & $17 / 02 / 2012$ & $20 / 02 / 2012$ & 3 & 814,20 & 53,70 \\
\hline 'SCSBRS Tio Taka' & $19 / 12 / 2011$ & $28 / 12 / 2011$ & $01 / 03 / 2012$ & $05 / 03 / 2012$ & 4 & 987,70 & 53,50 \\
\hline
\end{tabular}

Ciência Rural, v.46, n.3, mar, 2016. 
Tabela 2 - Data de ocorrência da iniciação da panícula (IP), da diferenciação da panícula (DP), diferença entre as datas de DP e IP, em dias, soma térmica acumulada (STA) para atingir a DP e diferença de STA entre as datas de DP e IP, em ${ }^{\circ} \mathrm{C}$ dia.

\begin{tabular}{|c|c|c|c|c|c|c|c|}
\hline Cultivar & $\begin{array}{c}\text { Data de } \\
\text { Semeadura }\end{array}$ & $\begin{array}{c}\text { Data de } \\
\text { Emergência }\end{array}$ & IP & DP & $\begin{array}{l}\text { DP-IP } \\
\text { (dias) }\end{array}$ & $\begin{array}{c}\text { STA } \\
\left({ }^{\circ} \mathrm{C} \text { dia }\right)\end{array}$ & $\begin{array}{c}\text { DP-IP } \\
\left({ }^{\circ} \mathrm{C} \text { dia }\right)\end{array}$ \\
\hline 'IRGA 421' & $19 / 10 / 2012$ & $29 / 10 / 2012$ & - & $03 / 12 / 2012$ & - & 439,50 & - \\
\hline 'BRS Querência' & 19/10/2012 & $29 / 10 / 2012$ & $10 / 12 / 2012$ & $13 / 12 / 2012$ & 3 & 578,70 & 36,80 \\
\hline 'IRGA 424' & $19 / 10 / 2012$ & $29 / 10 / 2012$ & $21 / 12 / 2012$ & $26 / 12 / 2012$ & 5 & 765,70 & 77,70 \\
\hline 'SCSBRS Tio Taka' & $19 / 10 / 2012$ & $29 / 10 / 2012$ & $04 / 01 / 2013$ & $10 / 01 / 2013$ & 6 & 943,60 & 77,90 \\
\hline 'IRGA 421' & $18 / 11 / 2012$ & $16 / 11 / 2012$ & $19 / 12 / 2012$ & $21 / 12 / 2012$ & 2 & 482,40 & 24,80 \\
\hline ‘BRS Querência' & $18 / 11 / 2012$ & $16 / 11 / 2012$ & $28 / 12 / 2012$ & $30 / 12 / 2012$ & 2 & 600,90 & 25,50 \\
\hline 'IRGA 424' & $18 / 11 / 2012$ & $16 / 11 / 2012$ & $07 / 01 / 2013$ & $11 / 01 / 2013$ & 4 & 748,50 & 51,50 \\
\hline 'SCSBRS Tio Taka' & $18 / 11 / 2012$ & $17 / 11 / 2012$ & $17 / 01 / 2013$ & $21 / 01 / 2013$ & 4 & 861,80 & 47,50 \\
\hline 'IRGA 421' & $04 / 01 / 2013$ & $15 / 01 / 2013$ & $22 / 02 / 2013$ & $26 / 02 / 2013$ & 4 & 555,20 & 48,60 \\
\hline 'BRS Querência' & $04 / 01 / 2013$ & $15 / 01 / 2013$ & $02 / 03 / 2013$ & $05 / 03 / 2013$ & 3 & 626,10 & 28,40 \\
\hline 'IRGA 424' & $04 / 01 / 2013$ & $15 / 01 / 2013$ & $12 / 03 / 2013$ & $15 / 03 / 2013$ & 3 & 724,30 & 23,80 \\
\hline 'SCSBRS Tio Taka' & $04 / 01 / 2013$ & $15 / 01 / 2013$ & $19 / 03 / 2013$ & $25 / 03 / 2013$ & 6 & 805,40 & 55,30 \\
\hline
\end{tabular}

ciclo muito precoce e precoce. Os resultados deste estudo não comprovaram essas hipóteses.

A aplicação prática dos resultados deste trabalho é que o manejo da adubação nitrogenada em cobertura pode ser melhorado, no sentido de que a segunda dose pode ser aplicada em R0 (IP), como recomendado pela SOSBAI (2012) e, assim, contribuir para um possível aumento na produtividade de arroz no Rio Grande do Sul. Para isso, o usuário pode estimar a data de DP (R1) pelo método de graus-dia proposto por STEINMETZ et al. (2010), baseado na série histórica (30 anos) de dados de temperatura média diária do ar (Tm), e corrigir a data de DP com a Tm do ano da safra por meio do programa disponível no endereço $<$ http://www.cpact.embrapa.br/agromet $>$, clicando em "Graus-dia (Arroz)" e em "Consulta por localidades". Ao dispor da data corrigida de DP, o usuário poderá antecipar em até 8 dias, pois compreenderá todas as cultivares que foram testadas neste experimento, e não terá risco de realizar a adubação nitrogenada fora do período recomendado pela SOSBAI.

\section{CONCLUSÃO}

O número médio de dias em que a iniciação da panícula (IP) antecede a diferenciação da panícula (DP) é de quatro dias, correspondendo a $53,1^{\circ} \mathrm{C}$ dia, não sendo influenciado pela época de semeadura nem pela duração do ciclo das cultivares de arroz. $\mathrm{O}$ período máximo que a IP antecede a DP é de oito dias, correspondendo a $116,4^{\circ} \mathrm{C}$ dia.

\section{AGRADECIMENTOS}

À Empresa Brasileira de Pesquisa Agropecuária (Embrapa) pela concessão de bolsa (estágio remunerado) ao autor Cristiano de Carli (Projeto MP3, código SEG 03.11.01.010.00.00) e ao Conselho Nacional de Desenvolvimento Científico e Tecnológico (CNPq, Proc. No 302524/2011-8) pelo apoio financeiro.

\section{REFERÊNCIAS}

CONAB. Acompanhamento da safra brasileira de grãos. Brasília: Companhia Nacional de Abastecimento, 2014. N.6, p.183. Online. Disponível em: <http://www.conab.gov.br/OlalaCMS/ uploads/arquivos/14_03_12_08_41_24_boletim_graos marco_2014.pdf $>$.Acesso em: 10 jun. 2014.

COUNCE, P.A. et al. A uniform, objective, and adaptive system for expressing rice development. Crop Science, Madison, v.40, n.2, p.436-443, mar./apr. 2000. Disponível em: <https:// dl.sciencesocieties.org/publications/cs/abstracts/40/2/436>. Acesso em: 19 mai. 2014. doi: 10.2135/cropsci2000.402436x.

FAO. Perspectivas iniciais para os cereais em 2014. Roma: Organização das Nações Unidas para a Agricultura e Alimentação, abr. 2014. Online. Disponível em: <http:/www.fao.org/news/ story/pt/item/219087/icode/>. Acesso em: 10 jun. 2014.

INFELD, J.A. et al. Temperatura-base e graus-dia durante o período vegetativo de três grupos de cultivares de arroz irrigado. Revista Brasileira de Agrometeorologia, Santa Maria, v.6, n.2, p.187-191, dez. 1998.

IRRI. Development stages of panicle formation to flowering. Rice Knowledge Bank: International Rice Research Institute. Online. 
Disponível em: <http://knowledgebank.cimmyt.org/extension/ hybridriceadjusting-flowering-date/hybridricedevelopmentstages-of-panicle-formation-to-flowering.html $>$. Acesso em: 28 abr. 2014.

MAPA. Portaria n $\mathbf{n}^{\mathbf{2}}$ 67, de 5 de dezembro de 2013. Brasília: Ministério da Agricultura Pecuária e Abastecimento, 2013. Online. Disponível em: <http://sistemasweb.agricultura.gov.br/sislegis/ action/detalhaAto.do?method=visualizarAtoPortalMapa\&cha $\mathrm{ve}=1841327206>$. Acesso em: 10 jun. 2014 .

SLATON, N.A. et al. Efficient use of fertilizer. In: HELMS, R.S. Rice production handbook. Little Rock: University of Arkansas, 1996. p.42-54.

SOSBAI. Arroz irrigado: recomendações técnicas de pesquisa para o Sul do Brasil. Itajaí: Sociedade Sul-Brasileira de Arroz Irrigado, 2012. 179p.

STANSEL, J.W. The rice plant: its development and yield. In: Six decades of rice research in Texas. Beaumont:
The Texas Agricultural Experiment Station, The Texas A\&M University System, 1975. p.9-21.

STEINMETZ, S. et al. Determinação dos graus-dia e do número de dias para atingir o estádio de diferenciação da panícula de cultivares de arroz irrigado. Pelotas: Embrapa Clima Temperado, 2009. 29p. (Boletim de pesquisa e desenvolvimento, 88).

STEINMETZ, S. et al. Uso de graus-dia para estimar a data de diferenciação da panícula (DP) de seis subgrupos de cultivares de arroz irrigado visando à adubação nitrogenada em cobertura no Rio Grande do Sul. Pelotas: Embrapa Clima Temperado, 2010. 75p. (Boletim de pesquisa e desenvolvimento, 121).

STEINMETZ, S. et al. GD arroz: programa baseado em grausdia para estimar a data de diferenciação da panícula visando a adubação nitrogenada em cobertura. Pelotas: Embrapa Clima Temperado, 2014. 12p. (Circular Técnica, 155).

YOSHIDA, S. Fundamentals of rice crop science. Manila: IRRI, 1981. 269p. 